Relations industrielles

Industrial Relations

\title{
The Miners: One Union, One Industry; A History of the National Union of Mineworkers, 1939-46 par R. Page Arnot, Londres, George Allen and Unwin, 1979, 212 pp.
}

\section{Trevor Burridge}

\section{Volume 35, numéro 1, 1980}

URI : https://id.erudit.org/iderudit/029054ar

DOI : https://doi.org/10.7202/029054ar

Aller au sommaire du numéro

Éditeur(s)

Département des relations industrielles de l'Université Laval

ISSN

0034-379X (imprimé)

1703-8138 (numérique)

Découvrir la revue

Citer ce compte rendu

Burridge, T. (1980). Compte rendu de [The Miners: One Union, One Industry; A History of the National Union of Mineworkers, 1939-46 par R. Page Arnot, Londres, George Allen and Unwin, 1979, 212 pp.] Relations industrielles / Industrial Relations, 35(1), 174-175. https://doi.org/10.7202/029054ar

Tous droits réservés (C) Département des relations industrielles de l'Université Laval, 1980
Ce document est protégé par la loi sur le droit d'auteur. L’utilisation des services d’Érudit (y compris la reproduction) est assujettie à sa politique d'utilisation que vous pouvez consulter en ligne.

https://apropos.erudit.org/fr/usagers/politique-dutilisation/ 
The Miners: One Union, One Industry; A History of the National Union of Mineworkers, 1939-46, par R. Page Arnot, Londres, George Allen and Unwin, 1979, 212 pp.

La seconde guerre mondiale s'est avérée un point tournant et décisif dans l'histoire du syndicalisme des mineurs britanniques. Car c'est pendant ces années là que leur organisation fédérale, fondée en 1889 , s'est transformée en organisation nationale. De plus, en 1946, le National Union of Mineworkers est venu s'incorporer au National Federation of Colliery Officials and Staffs, prévoyant ainsi le regroupement de tous les employés des mines de charbon, jusqu'aux sous-directeurs, en un seul syndicat.

C'est l'une des faiblesses, malheureusement typique de cet ouvrage, que la date exacte de la formation du N.U.M. ne soit citée nulle part. L'auteur, R. Page Arnot, est pourtant l'une des autorités reconnues sur le sujet; en effet, le présent livre constitue le quatrième volume de la série qu'il a consacrée à l'histoire de l'organisation syndicale centrale des mineurs. Mais il l'a entrepris pendant sa neuvième décennie et les signes d'une certaine fatigue sont évidents - recherche insuffisante, manque de cohésion, analyse plutôt superficielle et lassitude de style. Le drame de ces années lui ont largement échappé et, en outre, nous sommes ici dans le royaume de l'histoire officielle, sèche et souvent aride.

Néanmoins, les points saillants de l'affaire sont là pour ceux qui veulent les découvrir. En particulier, c'est l'effort de guerre qui avait exigé cette unification qui était accomplie dans le cadre de la centralisation de toutes les institutions économiques du pays. Mais comme toujours, le cas des mineurs était un peu spécial. Ce n'est qu'en 1942 que le gouvernement de coalition a mis en marche le système de "dual-control" sur l'industrie charbonnière, exigeant que les mineurs parlent d'une seule voix.

Malgré la perte de presque trente mille hommes aux services militaires pendant les premières semaines de la guerre, les événe- ments de la drôle de guerre ont mené à une augmentation du chômage parmi les mineurs. L'entrée de l'Italie dans la guerre et puis la chute de la France ont détruit une bonne partie du marché d'exportation. Il fallait l'hiver désastreux de 1941-42 pour que le nouveau gouvernement se rende compte de la nécessité cruciale d'une augmentation de la production charbonnière. À ce moment l'industrie était dans un état de désordre et de démoralisation. Plus de mille sociétés indépendantes essayaient de produire du charbon de presque deux mille puits. Et maintenant la difficulté majeure était devenue une pénurie de mineurs. Ainsi, en juin 1942, fut créé un nouveau département d'État, le Ministry of Fuel and Power, ayant le plein contrôle des opérations de tous les puits de charbon. Le ministre devait recevoir l'aide du National Coal Board, représentant les intérêts des mineurs et des propriétaires de mines. Donc, bon gré mal gré, les mineurs étaient obligés de se doter d'une organisation unique.

$\mathrm{Au}$ début de la guerre, les chefs de la Miners' Federation of Great Britain espéraient obtenir ce résultat par l'intermédiaire de la nationalisation de l'industrie. Ils ont continué à soutenir cette politique durant toute la guerre face à un gouvernement partagé et hésitant. Les ministres travaillistes étaient évidemment d'accord mais Churchill a toujours refusé. Le seul objet de la Coalition, selon lui, était la victoire; des modifications sociales et économiques d'une nature fondamentale à long terme devaient attendre la fin de la guerre et le retour de l'activité politique normale. Sa timidité en ce domaine a sans doute coûté à Churchill l'élection générale de 1945. Peu après l'élection, il avouait à un chef syndical que les propriétaires des mines étaient la meule qui avait fait couler le parti conservateur.

Quoi qu'il en soit, l'opposition du parti conservateur au projet de nationalisation du gouvernement Attlee n'était pas forte. L'un des porte-parole de l'opposition, Harold Macmillan, avait déjà préconisé la nationalisation de l'industrie charbonnière dans les années trente. La deuxième et plus médiocre 
section du livre de Page Arnot raconte la présentation du Bill au Parlement de 1945 à 1946. Son récit est fondé presque entièrement sur des extraits d'Hansard et n'ajoute rien aux détails connus. Mais l'un des arguments favoris des conservateurs allait prendre une signification ironique. "Vous n'allez que remplacer un groupe de patrons par un autre», - disaient-ils en se moquant des députés mineurs, "et choisir un patron plus éloigné et plus fort - l'état». L'avenir allait révéler la force de cette observation, et aussi sa faiblesse.

\section{Trevor BURRIDGE}

Université de Montréal

Labor Relations Law, 3rd edition, by B.J. Taylor and F. Witney, Prentice-Hall, Inc., 1979, 834 pp.

Fidele aux éditions antérieures, cet ouvrage n'en est pas un strictement juridique. Les auteurs étudient en effet, plus exactement présentent l'ordonnancement légal régissant la négociation collective aux États-Unis en le remplaçant dans son environnement. Le juriste comme le non-juriste peut donc encore trouver un intérêt certain dans ce véritable traité.

La structure de présentation n'a pas été modifiée. Ainsi, en dépit des six grands thèmes qui retiennent toujours l'attention, il nous semble que les auteurs privilégient trois axes fondamentaux dans l'évolution. Les premiers propos, qui comportent des données de mise en contexte, couvrent la période des origines jusqu'au Wagner Act (i: Introduction to Collective Agreement; ii: Legal Suppression of Collective Agreement; iii: Governement Encouragement to Collective Bargaining). Par la suite, le lecteur se voit offrir une revue des principaux événements en- tourant l'adoption et les lendemains du TaftHartley Act (iv: Control of Collective Bargaining; Collective bargaining: Area of Industrial Conflict). Finalement, il est question du Lendrum-Griffin Act jusqu'à nos jours (vi: Additional Areas of Government Control).

Si l'on fait exception d'un nouveau chapitre consacré à la discrimination dans l'emploi, incidemment des plus intéressant, le contenu n'a pas été substantiellement modifié. En fait, il s'agit d'une mise à jour où les auteurs font bien ressortir les nouvelles approches retenues en jurisprudence. On notera une exception; l'étude des relations du travail dans le secteur public, notamment dans les hôpitaux, a été à peu de chose près complètement reprise. Nous signalons la présentation des programmes de refonte du président Carter et des problèmes encourus. Notons finalement en fin du volume la reproduction des huit (8) principales lois américaines en la matière.

De façon générale, cet ouvrage conserve la qualité de ses prédécesseurs: clarté et concision. On notera que les auteurs ont fait un effort certain pour expliquer davantage les coûts de la négociation collective, des politiques publiques en relations du travail, de la "socio-economic rationale" des pièces législatives. Le but recherché semble d'amener le lecteur à mieux évaluer le système. La démarche peut susciter la réflexion.

Cet ouvrage mérite d'être parcouru et constitue indéniablement un instrument de réflexion qui doit se retrouver en bibliothèque.

Rodrigue BLOUIN

Université Laval 